\title{
On Fixed-Point Smoothing for Descriptor Systems with Multiplicative Noise and Single Delayed Observations
}

\author{
Xiao Lu, Xin Dong, Haixia Wang, and Baoye Song \\ Key Laboratory for Robot \& Intelligent Technology of Shandong Province, Shandong University of Science and Technology, \\ Qingdao 266590, China
}

Correspondence should be addressed to Xiao Lu; luxiao98@163.com

Received 9 January 2015; Revised 5 February 2015; Accepted 5 February 2015

Academic Editor: Kun Liu

Copyright (C) 2015 Xiao Lu et al. This is an open access article distributed under the Creative Commons Attribution License, which permits unrestricted use, distribution, and reproduction in any medium, provided the original work is properly cited.

Optimal fixed-point smoothing problem for the descriptor systems with multiplicative noises is considered, where instantaneous and delayed observations are available. Standard singular value decomposition is used to give the restricted equivalent delayed system, where the observations also include two different types of measurements. Reorganized innovation lemma and projection theorem are used to give the fixed-point smoother for the restricted equivalent delayed system. The fixed-point smoother is given in terms of recursive Riccati equations.

\section{Introduction}

The optimal estimation problem has long been one of the important problems in control theory and signal processing [1-4], and it is the dual problem of control [5-8]. Estimation includes three cases: prediction, filter, and smoothing [9-11], and smoothing problem is the most difficult among three problems. Smoothing problem is to estimate the past state or signal based on the observations in future, which mainly includes fixed-point smoothing, fixed-interval smoother, and fixed-lag smoother, where fixed-point is to estimate the fixedpoint state or signal in past based on its future observations, which can reveal the development trend of estimation with the increase of observations $[9,12,13]$. Under the optimal performance, estimation (prediction, filter, and smoothing) problem for normal system without multiplicative noises and delayed measurements has been studied well in recent years $[12,13]$.

The optimal smoothing problem has received much attention these years $[9,10,12,13]$. For the optimal smoothing of descriptor systems with multiplicatives, some researchers have given some important results [14-16], where Kalman filtering and standard decomposition are to study the optimal estimation of the descriptor systems.

As is well known, the measured output may be with delay in practical applications such as engineering, biological, and economic systems [8]. However, the above descriptor systems are without delay in observation; for the systems with delayed measurement, the classic approach is system or variable augmentation [11, 17], which may lead to much computation burden. In this paper, the optimal fixed-point smoothing problems for descriptor systems with multiplicative noises and delayed measured output will be studied. Being different from the classic system augmentation, the reorganized innovation lemma developed in our previous works $[17,18]$ will be proposed. Standard singular value decomposition will be used to change the system into the restricted equivalent delayed system.

The presented approach is very efficient and important for estimation on descriptor system with multiplicative noise, and reorganized innovation lemma is used to decrease much computation burden compared to traditional system augmentation [17]. The proposed result extends the optimal filter and multistep predictor [19].

The rest of the paper is organized as follows. The fixedpoint smoothing problem will be proposed in Section 2. Substate fixed-point smoother will be given for the restricted equivalent delayed system in Section 3. Fixed-point smoother for the delayed descriptor systems with multiplicative noise will be given in Section 4. Some concluding remarks will be drawn in Section 5. 


\section{Problem Statement}

In this paper, we will deal with the following linear discretetime descriptor system:

$$
\begin{gathered}
F \mathbf{x}(t+1)=A \mathbf{x}(t)+B \mathbf{u}(t), \\
\mathbf{y}(t)=C \mathbf{x}(t)+D \mathbf{w}(t) \mathbf{x}(t)+\mathbf{v}(t), \\
\mathbf{y}_{d}(t)=C_{d} \mathbf{x}\left(t_{d}\right)+D_{d} \mathbf{w}(t) \mathbf{x}\left(t_{d}\right)+\mathbf{v}_{d}(t), \quad t_{d}=t-d,
\end{gathered}
$$

where $A, B, C, D, C_{d}$, and $D_{d}$ are known matrices and the information of other parameters can be listed as

$$
\begin{aligned}
& d \rightarrow R^{1} \rightarrow \text { delay, } \\
& \mathbf{x}(t) \rightarrow R^{n} \rightarrow \text { state, } \\
& \mathbf{y}(t), \mathbf{v}(t) \rightarrow R^{q} \rightarrow \text { observation and its noise, } \\
& \begin{array}{l}
\mathbf{y}_{d}(t), \mathbf{v}_{d}(t) \rightarrow R^{q_{d}} \rightarrow \text { delayed observation } \\
\text { noise, }
\end{array} \\
& \mathbf{u}(t) \rightarrow R^{p} \rightarrow \text { input disturbance, } \\
& \mathbf{w}(t) \rightarrow R^{1} \rightarrow \text { multiplicative noise. }
\end{aligned}
$$$$
\mathbf{y}_{d}(t), \mathbf{v}_{d}(t) \rightarrow R^{q_{d}} \rightarrow \text { delayed observation and its }
$$

We first give two assumptions as follows.

Assumption 1. $\mathbf{u}(t), \mathbf{v}(t), \mathbf{v}_{d}(t)$, and $\mathbf{w}(t)$ are uncorrelated white noises of zero means and uncorrelated with $\mathbf{x}(0)$, and the corresponding variance is $\langle\boldsymbol{\xi}(t), \boldsymbol{\xi}(s)\rangle=Q_{\xi} \delta_{t, s}$, and $\xi(t)$ can be $\mathbf{u}(t), \mathbf{v}(t), \mathbf{v}_{d}(t)$, and $\mathbf{w}(t)$; in addition, $\langle\mathbf{x}(0), \mathbf{x}(0)\rangle=$ $\Pi(0)$.

Assumption 2. F is known and singular, and the system is regular, that is, $\operatorname{rank} F=n_{1}<n$, and there exists $s$ that satisfies $\operatorname{det}(s F-A) \neq 0$.

Remark 3. Assumption 1 is given for general optimal or $\mathrm{H}_{2}$ estimation problem. Assumption 2 is standard assumption for general descriptor system, since the regularity is very important for the existence of solution which is dependent on the initial value for descriptor system $[14,20]$.

Optimal fixed-point smoothing (FPS) problem for the above descriptor system model (1) can be described as follows.

Problem FPS. Consider the system model (1) with the instantaneous and delayed observations $\left\{\mathbf{y}(0), \ldots, \mathbf{y}(k) ; \mathbf{y}_{d}(d), \ldots\right.$, $\left.\mathbf{y}_{d}(k)\right\}$, a fixed time $t$ and $t \leq k \leq N$; find the linear least square error smoother $\widehat{\mathbf{x}}(t \mid k)$ of $\mathbf{x}(t)$, where $0 \leq t \leq N$.

Under Assumption 2, according to the classical result of descriptor system $[11,14,20]$, there exist nonsingular matrices $\left\{J_{1}, J_{2}\right\} \in R^{n \times n}, \mathbf{x}(t)=J_{2}\left[\begin{array}{l}\mathbf{x}_{1}(t) \\ \mathbf{x}_{2}(t)\end{array}\right]$, and we can give the following lemma.
Lemma 4 (see [19]). System (1) under Assumptions 1 and 2 can be restricted equivalent to

$$
\begin{gathered}
\mathbf{x}_{1}(t+1)=A_{1} \mathbf{x}_{1}(t)+B_{1} \mathbf{u}(t), \\
F_{1} \mathbf{x}_{2}(t+1)=\mathbf{x}_{2}(t)+B_{2} \mathbf{u}(t), \\
\mathbf{y}(t)=C_{1} \mathbf{x}_{1}(t)+C_{2} \mathbf{x}_{2}(t)+D_{1} \mathbf{w}(t) \mathbf{x}_{1}(t) \\
+D_{2} \mathbf{w}(t) \mathbf{x}_{2}(t)+\mathbf{v}(t), \\
\mathbf{y}_{d}(t)=C_{1 d} \mathbf{x}_{1}\left(t_{d}\right)+C_{2 d} \mathbf{x}_{2}\left(t_{d}\right)+D_{1 d} \mathbf{w}(t) \mathbf{x}_{1}\left(t_{d}\right) \\
+D_{2 d} \mathbf{w}(t) \mathbf{x}_{2}\left(t_{d}\right)+\mathbf{v}_{d}(t),
\end{gathered}
$$

where

$$
\begin{gathered}
J_{1} F J_{2}=\left[\begin{array}{cc}
I_{n_{1}} & 0 \\
0 & F_{1}
\end{array}\right], \quad J_{1} A J_{2}=\left[\begin{array}{cc}
A_{1} & 0 \\
0 & I_{n-n_{1}}
\end{array}\right], \\
J_{1} B=\left[\begin{array}{l}
B_{1} \\
B_{2}
\end{array}\right], \quad\left[\begin{array}{c}
C \\
C_{d} \\
D \\
D_{d}
\end{array}\right] J_{2}=\left[\begin{array}{cc}
C_{1} & C_{2} \\
C_{1 d} & C_{2 d} \\
D_{1} & D_{2} \\
D_{1 d} & D_{2 d}
\end{array}\right],
\end{gathered}
$$

$\mathbf{x}_{1} \in R^{n_{1}}, \mathbf{x}_{2} \in R^{n-n_{1}}$, and $F_{1}$ is a $\lambda$-nilpotent matrix; that is, $F_{1}^{\lambda}=0, F_{1}^{\lambda-1} \neq 0$.

\section{Optimal Fixed-Point Smoother $\widehat{\mathbf{x}}_{1}(t \mid k)$}

From Lemma 4, in order to give the fixed-point smoother of Problem FPS, we first give the fixed-point smoother of the restricted equivalent system (2)-(5).

3.1. Riccati Equation $P_{1}(t+1, i)$. The Riccati equation will be given mainly by using reorganized innovation analysis [17], the corresponding definitions $\mathbf{e}_{1}(t, i), \widetilde{\mathscr{Y}}_{i}(t), \Pi_{1}(t), P_{1}(t, i)$, $P_{1}(t, s, i), L(t, s, i), Q_{\bar{y}_{i}}(t), T(t, s, i), R(t, s, i)$, and $R(t, s, 12)$ in [19], and the following denotations:

$$
\left[\begin{array}{llll}
G(t, s, i) & G_{1}(t, s, i) & G_{2}(t, s \mid l, i) & G_{3}(t, s \mid l, i) \\
H(t, s, i) & H_{1}(t, s, i) & H_{2}(t, s \mid l, i) & H_{3}(t, s \mid l, i)
\end{array}\right]
$$

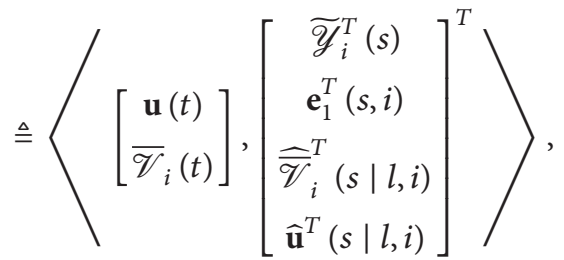

$$
\left[H(t, s, 12) \quad H_{1}(t, s, 12) \quad H_{2}(t, s \mid l, 12) \quad H_{3}(t, s \mid l, 12)\right]
$$

$$
\triangleq\left\langle\overline{\mathscr{V}}_{1}(t),\left[\begin{array}{c}
\widetilde{\mathscr{Y}}_{2}^{T}(s) \\
\mathbf{e}_{1}^{T}(s, 2) \\
\widehat{\mathscr{V}}_{2}^{T}(s \mid l, 2) \\
\widehat{\mathbf{u}}^{T}(s \mid l, 2)
\end{array}\right]^{T}\right\rangle .
$$

$P_{1}(t+1,1)$ can be given in the following lemma. 
Lemma 5 (see [19]). Consider the restricted equivalent delayed descriptor system (2)-(5) under Assumptions 1 and 2; the error covariance matrix $P_{1}(t+1,1)$ of $\mathbf{x}_{1}(t+1)$ can be given as

$$
\begin{aligned}
& P_{1}(t+1,1)= A_{1} P_{1}(t, 1) A_{1}^{T}+B_{1}\left[Q_{u}-G_{3}(t, t \mid t, 1)\right] B_{1}^{T} \\
&+A_{1}\left[G_{1}^{T}(t, t, 1)-K(t, t, 1) G^{T}(t, t, 1)\right] B_{1}^{T} \\
&+B_{1}\left[G_{1}(t, t, 1)-G(t, t, 1) K^{T}(t, t, 1)\right] A_{1}^{T} \\
&-A_{1} K(t, t, 1) Q_{\widetilde{Y}_{1}}(t) K^{T}(t, t, 1) A_{1}^{T}, \\
& P_{1}\left(t_{d}+1,1\right)=P_{1}\left(t_{d}+1,2\right), \quad t \geq d,
\end{aligned}
$$

where

$$
K(t, t, 1)=L(t, t, 1) Q_{\widetilde{Y}_{1}}^{-}(t)
$$

with

$$
\begin{aligned}
& L(t, t, 1)=P_{1}(t, 1) \mathscr{C}_{11}^{T}+H_{1}^{T}(t, t, 1) \\
& Q_{\widetilde{\mathscr{Y}}_{1}}(t)= \mathscr{C}_{11} P_{1}(t, 1) \mathscr{C}_{11}^{T}+D_{1} M \Pi_{1}(t) D_{1}^{T}+R(t, t, 1) \\
&-H_{2}(t, t \mid t-1,1)+\mathscr{C}_{11} H_{1}^{T}(t, t, 1) \\
&+H_{1}(t, t, 1) \mathscr{C}_{11}^{T},
\end{aligned}
$$

where

$$
\begin{aligned}
R(t, t, 1)= & Q_{v}+\sum_{j=0}^{\lambda-1} \mathscr{C}_{21} F_{1}^{j} B_{2} Q_{u} B_{2}^{T}\left(F_{1}^{j}\right)^{T} \mathscr{C}_{21}^{T} \\
& +M \sum_{j=0}^{\lambda-1} D_{2} F_{1}^{j} B_{2} Q_{u} B_{2}^{T}\left(F_{1}^{j}\right)^{T} D_{2}^{T},
\end{aligned}
$$

$G(t, t, 1), G_{1}(t, t, 1), G_{3}(t, t \mid t, 1), H_{1}(t, t, 1)$, and $H_{2}(t, t \mid$ $t-1,1)$ are defined in (7) and given in [19], and $\Pi_{1}(t)=$ $A_{1} \Pi_{1}(t) A_{1}^{T}+B_{1} Q_{u} B_{1}^{T}$.

3.2. Fixed-Point Smoother $\widehat{\mathbf{x}}_{1}(t \mid k)$. In this subsection, we will give the optimal fixed-point smoother for $\mathbf{x}_{1}(t)(k=t+$ $1, \ldots, N)$ based on the above lemmas.

Theorem 6. For a fixed $t$, consider the presented restricted equivalent delayed system (2)-(5) under Assumptions 1 and 2; the fixed-point smoother $\widehat{\mathbf{x}}_{1}(t \mid k)$ can be given as

$$
\begin{aligned}
& \widehat{\mathbf{x}}_{1}(t \mid k)= \widehat{\mathbf{x}}_{1}(t \mid t, 1)+\sum_{j=1}^{k-t} L(t, t+j, 1) Q_{\widetilde{\mathscr{Y}}_{1}}^{-}(t+j) \\
& \times\left[\mathscr{Y}_{1}(t+j)-\mathscr{C}_{11} \widehat{\mathbf{x}}_{1}(t+j, 1)-\widehat{\overline{\mathscr{V}}}_{1}(t+j, 1)\right], \\
& k=t+1, \ldots, N, \quad t \geq d,
\end{aligned}
$$

where

$$
\begin{aligned}
& \widehat{\mathbf{x}}_{1}(t \mid t, 1) \\
& =\widehat{\mathbf{x}}_{1}(t, 1) \\
& +L(t, t, 1) Q_{\widetilde{\mathscr{Y}}_{1}}^{-}(t)\left[\mathscr{Y}_{1}(t)-\mathscr{C}_{11} \widehat{\mathbf{x}}_{1}(t, 1)-\widehat{\overline{\mathscr{V}}}_{1}(t, 1)\right], \\
& L(t, t+j, 1) \\
& =P_{1}(t, t+j, 1) \mathscr{C}_{11}^{T}+H_{1}^{T}(t+j, t, 1) \\
& -\sum_{i=0}^{j-1} L(t, t+i, 1) Q_{\widetilde{\mathscr{Y}}_{1}}^{-}(t+i) H^{T}(t+j, t+i, 1), \\
& j=1, \ldots, k-t
\end{aligned}
$$

with

$$
\begin{aligned}
P_{1}(t, t+j, 1) \triangleq & \left\langle\mathbf{e}_{1}(t, 1), \mathbf{e}_{1}(t+j, 1)\right\rangle=P_{1}(t, 1)\left(A_{1}^{j}\right)^{T} \\
& +\sum_{i=0}^{j-1} G_{1}^{T}(t+i, t, 1) B_{1}^{T}\left(A_{1}^{j-1-i}\right)^{T} \\
& -\sum_{i=0}^{j-1} \sum_{m=0}^{i} L(t, t+m, 1) Q_{\widetilde{\mathscr{V}}_{1}}^{-}(t+m) G^{T} \\
& -\sum_{i=0}^{j-1} L(t, t+j-1-i, 1) Q_{\widetilde{Y}_{1}}^{-}(t+j-1-i) \\
& \times L^{T}(t, t+j-1-i, 1)\left(A_{1}^{i+1}\right)^{T} .
\end{aligned}
$$

$L(t, t, 1)$ is as in (11); $P_{1}(t, 1)$ is as in (9); $\widehat{\mathbf{x}}_{1}(t, 1)$ and $\widehat{\mathbf{x}}_{1}(t+j, 1)$ $(j=1, \ldots, k-t)$ can be given as

$$
\begin{aligned}
& \widehat{\mathbf{x}}_{1}(t+1,1) \\
&= A_{1} \widehat{\mathbf{x}}_{1}(t, 1)+A_{1} L(t, t, 1) Q_{\widetilde{\mathscr{V}}_{1}}^{-}(t) \\
& \cdot\left[\mathscr{Y}_{1}(t)-\mathscr{C}_{11} \widehat{\mathbf{x}}_{1}(t, 1)-\widehat{\mathscr{V}}_{1}(t, 1)\right] \\
&+B_{1} \widehat{\mathbf{u}}(t \mid t, 1), \\
& \widehat{\mathbf{x}}_{1}\left(t_{d}+1,1\right)=\widehat{\mathbf{x}}_{1}\left(t_{d}+1,2\right),
\end{aligned}
$$$$
t \geq d
$$ 
and $\widehat{\mathbf{x}}_{1}\left(t_{d}+1,2\right)$ can be given by

$$
\begin{aligned}
& \widehat{\mathbf{x}}_{1}\left(t_{d}+1,2\right) \\
&= A_{1} \widehat{\mathbf{x}}_{1}\left(t_{d}, 2\right)+A_{1} L\left(t_{d}, t_{d}, 2\right) Q_{\widetilde{\mathscr{V}}_{2}}^{-}\left(t_{d}\right) \\
& \cdot\left[\mathscr{Y}_{2}\left(t_{d}\right)-\mathscr{C}_{12} \widehat{\mathbf{x}}_{1}\left(t_{d}, 2\right)-\widehat{\mathscr{V}}_{2}\left(t_{d}, 2\right)\right] \\
&+B_{1} \widehat{\mathbf{u}}\left(t_{d} \mid t_{d}, 2\right), \\
& \widehat{\mathbf{x}}_{1}(0,2)=\widehat{\mathbf{x}}_{1}(0 \mid-1,2)=0 .
\end{aligned}
$$

In the above,

$$
\begin{aligned}
\widehat{\mathbf{u}}(t \mid t, 1)= & \widehat{\mathbf{u}}(t \mid t-1,1)+G(t, t, 1) Q_{\widetilde{\mathscr{V}}_{1}}^{-}(t) \\
& \cdot\left[\mathscr{Y}_{1}(t)-\mathscr{C}_{11} \widehat{\mathbf{x}}_{1}(t, 1)-\widehat{\mathscr{\mathscr { V }}}_{1}(t, 1)\right], \\
\widehat{\mathbf{u}}\left(t_{d} \mid t_{d}, 2\right)= & \widehat{\mathbf{u}}\left(t_{d} \mid t_{d}-1,2\right)+G\left(t_{d}, t_{d}, 2\right) Q_{\mathscr{\mathscr { V }}_{2}}^{-}\left(t_{d}\right) \\
& \cdot\left[\mathscr{Y}_{2}\left(t_{d}\right)-\mathscr{C}_{12} \widehat{\mathbf{x}}_{1}\left(t_{d}, 2\right)-\widehat{\mathscr{V}}_{2}\left(t_{d}, 2\right)\right],
\end{aligned}
$$

where

$$
\begin{aligned}
& \widehat{\overline{\mathscr{V}}}_{1}(t, 1) \\
& =\widehat{\overline{\mathscr{V}}}_{1}(t \mid t-2,1)+H(t, t-1,1) Q_{\widetilde{\mathscr{V}}_{1}}^{-}(t-1) \\
& \times\left[\mathscr{Y}_{1}(t-1)-\mathscr{C}_{11} \widehat{\mathbf{x}}_{1}(t-1,1)-\widehat{\overline{\mathscr{V}}}_{1}(t-1,1)\right], \\
& \widehat{\overline{\mathscr{V}}}_{1}\left(t_{d}+1,1\right)=\widehat{\overline{\mathscr{V}}}_{1}\left(t_{d}+1,2\right),
\end{aligned}
$$$$
\widehat{\widehat{\mathscr{V}}}_{2}\left(t_{d}, 2\right)
$$$$
=\widehat{\overline{\mathscr{V}}}_{2}\left(t_{d} \mid t_{d}-2,2\right)+H\left(t_{d}, t_{d}-1,2\right) Q_{\widetilde{\mathscr{V}}_{2}}^{-}\left(t_{d}-1\right)
$$$$
\times\left[\mathscr{Y}_{2}\left(t_{d}-1\right)-\mathscr{C}_{12} \widehat{\mathbf{x}}_{1}\left(t_{d}-1,2\right)-\widehat{\overline{\mathscr{V}}}_{2}\left(t_{d}-1,2\right)\right],
$$$$
\widehat{\overline{\mathscr{V}}}_{2}(0,2)=0 \text {, }
$$

with $\widehat{\overline{\mathscr{V}}}_{1}\left(t_{d}+1,2\right)$ being given as

$$
\begin{aligned}
\widehat{\overline{\mathscr{V}}}_{1}\left(t_{d}+1,2\right) \\
=\widehat{\overline{\mathscr{V}}}_{1}\left(t_{d}+1 \mid t_{d}-1,2\right)+H\left(t_{d}+1, t_{d}, 12\right) Q_{\widetilde{\mathscr{V}}_{2}}^{-}\left(t_{d}\right) \\
\quad \times\left[\mathscr{Y}_{2}\left(t_{d}\right)-\mathscr{C}_{12} \widehat{\mathbf{x}}_{1}\left(t_{d}, 2\right)-\widehat{\overline{\mathscr{V}}}_{2}\left(t_{d}, 2\right)\right]
\end{aligned}
$$

Proof. According to the projection theorem, we have

$$
\begin{aligned}
& \widehat{\mathbf{x}}_{1}(t \mid N) \\
& =\operatorname{Proj}\left\{\mathbf{x}_{1}(t) \mid \widetilde{\mathscr{Y}}_{2}(0), \ldots, \widetilde{\mathscr{Y}}_{2}\left(t_{d}\right) ;\right. \\
& \left.\widetilde{\mathscr{Y}}_{1}\left(t_{d}+1\right), \ldots, \widetilde{\mathscr{Y}}_{1}(t), \ldots, \widetilde{\mathscr{Y}}_{1}(N)\right\} \\
& =\widehat{\mathbf{x}}_{1}(t \mid t)+\sum_{j=1}^{N-t} \operatorname{Proj}\left\{\mathbf{x}_{1}(t) \mid \widetilde{\mathscr{Y}}_{1}(t+j)\right\} \\
& =\widehat{\mathbf{x}}_{1}(t \mid t)+\sum_{j=1}^{N-t} L(t, t+j, 1) Q_{\widetilde{\mathscr{Y}}_{1}}^{-}(t+j) \widetilde{\mathscr{Y}}_{1}(t+j),
\end{aligned}
$$

which is (14).

Since $\widehat{\mathbf{x}}_{1}(t, 1) \perp \widetilde{\mathscr{Y}}_{1}(t+1)$, then

$$
\begin{aligned}
L(t, t+1,1)= & \left\langle\mathbf{x}_{1}(t), \widetilde{\mathscr{Y}}_{1}(t+1)\right\rangle \\
= & \left\langle\mathbf{e}_{1}(t, 1), \widetilde{\mathscr{Y}}_{1}(t+1)\right\rangle \\
= & P_{1}(t, t+1,1) \mathscr{C}_{11}^{T}+H_{1}^{T}(t+1, t, 1) \\
& -\left\langle\mathbf{e}_{1}(t, 1), \widehat{\mathscr{V}}_{1}(t+1,1)\right\rangle .
\end{aligned}
$$

According to the projection theorem, we have

$$
\begin{aligned}
& \widehat{\overline{\mathscr{V}}}_{1}(t+1,1) \\
& =\operatorname{Proj}\left\{\overline{\mathscr{V}}_{1}(t+1) \mid \widetilde{\mathscr{Y}}_{2}(0), \ldots, \widetilde{\mathscr{Y}}_{2}\left(t_{d}\right) ;\right. \\
& \left.\widetilde{\mathscr{Y}}_{1}\left(t_{d}+1\right), \ldots, \widetilde{\mathscr{Y}}_{1}(t)\right\} \\
& =\operatorname{Proj}\left\{\overline{\mathscr{V}}_{1}(t+1) \mid \widetilde{\mathscr{Y}}_{2}(0), \ldots, \widetilde{\mathscr{Y}}_{2}\left(t_{d}\right)\right. \text {; } \\
& \left.\widetilde{\mathscr{Y}}_{1}\left(t_{d}+1\right), \ldots, \widetilde{\mathscr{Y}}_{1}(t-1)\right\} \\
& +\operatorname{Proj}\left\{\overline{\mathscr{V}}_{1}(t+1) \mid \widetilde{\mathscr{Y}}_{1}(t)\right\} \\
& =\widehat{\overline{\mathscr{V}}}_{1}(t+1 \mid t-1,1)+H(t+1, t, 1) Q_{\widetilde{\mathscr{V}}_{1}}^{-}(t) \widetilde{\mathscr{Y}}_{1}(t) \text {, }
\end{aligned}
$$

which is (22). By considering $\mathbf{e}_{1}(t, 1) \perp \widehat{\mathscr{V}}_{1}(t+1 \mid t-1,1)$ and (27), (26) can be rewritten as

$$
\begin{aligned}
L(t, t+1,1)= & P_{1}(t, t+1,1) \mathscr{C}_{11}^{T}+H_{1}^{T}(t+1, t, 1) \\
& -L(t, t, 1) Q_{\widetilde{\mathscr{Y}}_{1}}^{-}(t) H^{T}(t+1, t, 1) .
\end{aligned}
$$

Similarly,

$$
\begin{aligned}
L(t, t+2,1)= & \left\langle\mathbf{e}_{1}(t, 1), \widetilde{\mathscr{Y}}_{1}(t+2)\right\rangle \\
= & P_{1}(t, t+2,1) \mathscr{C}_{11}^{T}+H_{1}^{T}(t+2, t, 1) \\
& -\left\langle\mathbf{e}_{1}(t, 1), \widehat{\mathscr{V}}_{1}(t+2,1)\right\rangle,
\end{aligned}
$$


while

$$
\begin{aligned}
\widehat{\mathscr{V}}_{1}(t+2,1)= & \widehat{\mathscr{V}}_{1}(t+2 \mid t, 1) \\
& +H(t+2, t+1,1) Q_{\widetilde{\mathscr{Y}}_{1}}^{-}(t+1) \widetilde{\mathscr{Y}}_{1}(t+1) \\
= & \widehat{\mathscr{V}}_{1}(t+2 \mid t-1,1) \\
& +H(t+2, t, 1) Q_{\widetilde{\mathscr{Y}}_{1}}^{-}(t) \widetilde{\mathscr{Y}}_{1}(t) \\
& +H(t+2, t+1,1) Q_{\overline{\mathscr{Y}}_{1}}^{-}(t+1) \widetilde{\mathscr{Y}}_{1}(t+1),
\end{aligned}
$$

since $\mathbf{e}_{1}(t, 1) \perp \widehat{\mathscr{V}}_{1}(t+2 \mid t-1,1)$, so from (29) and (30),

$$
\begin{aligned}
L(t, t+2,1)= & P_{1}(t, t+2,1) \mathscr{C}_{11}^{T}+H_{1}^{T}(t+2, t, 1) \\
& -L(t, t, 1) Q_{\widetilde{\mathscr{Y}}_{1}}^{-}(t) H^{T}(t+2, t, 1) \\
& -L(t, t+1,1) Q_{\widetilde{\mathscr{Y}}_{1}}^{-}(t+1) H^{T} \\
& \cdot(t+2, t+1,1) .
\end{aligned}
$$

Then by inductive method, we have

$$
\begin{array}{r}
L(t, t+j, 1) \\
=P_{1}(t, t+j, 1) \mathscr{C}_{11}^{T}+H_{1}^{T}(t+j, t, 1) \\
-\sum_{i=0}^{j-1} L(t, t+i, 1) Q_{\overline{\mathscr{Y}}_{1}}^{-}(t+i) H^{T}(t+j, t+i, 1), \\
\quad j=1, \ldots, k-t,
\end{array}
$$

which is (16).

According to the projection theorem, we have

$$
\begin{aligned}
& \widehat{\mathbf{x}}_{1}(t \mid t, 1) \\
& =\operatorname{Proj}\left\{\mathbf{x}_{1}(t) \mid \widetilde{\mathscr{Y}}_{2}(0), \ldots, \widetilde{\mathscr{Y}}_{2}\left(t_{d}\right) ;\right. \\
& \left.\widetilde{\mathscr{Y}}_{1}\left(t_{d}+1\right), \ldots, \widetilde{\mathscr{Y}}_{1}(t)\right\} \\
& =\widehat{\mathbf{x}}_{1}(t \mid t-1,1)+\operatorname{Proj}\left\{\mathbf{x}_{1}(t) \mid \widetilde{\mathscr{Y}}_{1}(t)\right\} \\
& =\widehat{\mathbf{x}}_{1}(t \mid t-1,1)+L(t, t, 1) Q_{\widetilde{\mathscr{Y}}_{1}}^{-}(t) \widetilde{\mathscr{Y}}_{1}(t) ;
\end{aligned}
$$

then (15) can be given.

Similarly, we have

$$
\begin{aligned}
& \widehat{\mathbf{x}}_{1}(t+1,1) \\
& =\operatorname{Proj}\left\{\mathbf{x}_{1}(t+1) \mid \widetilde{\mathscr{Y}}_{2}(0), \ldots, \widetilde{\mathscr{Y}}_{2}\left(t_{d}\right) ;\right. \\
& \left.\widetilde{\mathscr{Y}}_{1}\left(t_{d}+1\right), \ldots, \widetilde{\mathscr{Y}}_{1}(t)\right\} \\
& =\operatorname{Proj}\left\{A_{1} \mathbf{x}_{1}(t)+B_{1} \mathbf{u}(t) \mid \widetilde{\mathscr{Y}}_{1}(t)\right\} \\
& =A_{1} \widehat{\mathbf{x}}_{1}(t, 1)+A_{1} L(t, t, 1) Q_{\widetilde{\mathscr{V}}_{1}}^{-}(t) \widetilde{\mathscr{Y}}_{1}(t)+B_{1} \widehat{\mathbf{u}}(t \mid t, 1),
\end{aligned}
$$

which is (18). Then combining (34) with (2) yields

$$
\begin{aligned}
\mathbf{e}_{1}(t+1,1)= & A_{1} \mathbf{e}_{1}(t, 1)+B_{1} \mathbf{u}(t)-B_{1} \widehat{\mathbf{u}}(t \mid t, 1) \\
& -A_{1} L(t, t, 1) Q_{\widetilde{\mathscr{Y}}_{1}}^{-}(t) \widetilde{\mathscr{Y}}_{1}(t) .
\end{aligned}
$$

We have

$$
\begin{aligned}
\mathbf{e}_{1}(t+j, 1)= & A_{1}^{j} \mathbf{e}_{1}(t, 1)+\sum_{i=0}^{j-1} A_{1}^{j-1-i} B_{1} \mathbf{u}(t+i) \\
& -\sum_{i=0}^{j-1} A_{1}^{j-1-i} B_{1} \widehat{\mathbf{u}}(t+i \mid t+i, 1) \\
& -\sum_{i=0}^{j-1} A_{1}^{i+1} L(t, t+j-1-i, 1) Q_{\widetilde{\mathscr{Y}}_{1}^{-}} \\
& \cdot(t+j-1-i) \widetilde{\mathscr{Y}}_{1}(t+j-1-i) .
\end{aligned}
$$

According to the projection theorem, we have

$$
\begin{aligned}
& \widehat{\mathbf{u}}(t \mid t, 1) \\
& =\operatorname{Proj}\left\{\mathbf{u}(t) \mid \widetilde{\mathscr{Y}}_{2}(0), \ldots, \widetilde{\mathscr{Y}}_{2}\left(t_{d}\right) ;\right. \\
& \left.\widetilde{\mathscr{Y}}_{1}\left(t_{d}+1\right), \ldots, \widetilde{\mathscr{Y}}_{1}(t)\right\} \\
& =\operatorname{Proj}\left\{\mathbf{u}(t) \mid \widetilde{\mathscr{Y}}_{2}(0), \ldots, \widetilde{\mathscr{Y}}_{2}\left(t_{d}\right) ;\right. \\
& \left.\quad \widetilde{\mathscr{Y}}_{1}\left(t_{d}+1\right), \ldots, \widetilde{\mathscr{Y}}_{1}(t-1)\right\} \\
& \quad+\operatorname{Proj}\left\{\mathbf{u}(t) \mid \widetilde{\mathscr{Y}}_{1}(t)\right\} \\
& =\widehat{\mathbf{u}}(t \mid t-1,1)+\left\langle\mathbf{u}(t), \widetilde{\mathscr{Y}}_{1}(t)\right\rangle Q_{\widetilde{\mathscr{Y}}_{1}}(t) \widetilde{\mathscr{Y}}_{1}(t) \\
& =\widehat{\mathbf{u}}(t \mid t-1,1)+G(t, t, 1) Q_{\widetilde{\mathscr{Y}}_{1}}(t) \widetilde{\mathscr{Y}}_{1}(t) ;
\end{aligned}
$$

then (20) can be given.

Similarly,

$$
\begin{aligned}
\widehat{\mathbf{u}}(t+ & i \mid t+i, 1) \\
= & \widehat{\mathbf{u}}(t+i \mid t-1,1) \\
& +\sum_{m=0}^{i} G(t+i, t+m, 1) Q_{\widetilde{\mathscr{Y}}_{1}}^{-}(t+m) \widetilde{\mathscr{Y}}_{1}(t+m),
\end{aligned}
$$


since $\mathbf{e}_{1}(t, 1) \perp \widehat{\mathbf{u}}(t+i \mid t-1,1)$, so from (36) and (38), we have

$$
\begin{aligned}
& P_{1}(t, t+j, 1) \\
& =\left\langle\mathbf{e}_{1}(t, 1), \mathbf{e}_{1}(t+j, 1)\right\rangle \\
& =\left\langle\mathbf{e}_{1}(t, 1), A_{1}^{j} \mathbf{e}_{1}(t, 1)+\sum_{i=0}^{j-1} A_{1}^{j-1-i} B_{1} \mathbf{u}(t+i)\right. \\
& -\sum_{i=0}^{j-1} A_{1}^{j-1-i} B_{1} \widehat{\mathbf{u}}(t+i \mid t+i, 1) \\
& -\sum_{i=0}^{j-1} A_{1}^{i+1} L(t, t+j-1-i, 1) Q_{\overline{\mathscr{Y}}_{1}}^{-} \\
& \left.\cdot(t+j-1-i) \widetilde{\mathscr{Y}}_{1}(t+j-1-i)\right\rangle \\
& =P_{1}(t, 1)\left(A_{1}^{j}\right)^{T}+\sum_{i=0}^{j-1} G_{1}^{T}(t+i, t, 1) B_{1}^{T}\left(A_{1}^{j-1-i}\right)^{T} \\
& -\sum_{i=0}^{j-1} \sum_{m=0}^{i} L(t, t+m, 1) Q_{\mathscr{\mathscr { V }}_{1}}^{-}(t+m) G^{T} \\
& \cdot(t+i, t+m, 1) B_{1}^{T}\left(A_{1}^{j-1-i}\right)^{T} \\
& -\sum_{i=0}^{j-1} L(t, t+j-1-i, 1) Q_{\widetilde{\mathscr{Y}}_{1}}^{-}(t+j-1-i) L^{T} \\
& \cdot(t, t+j-1-i, 1)\left(A_{1}^{i+1}\right)^{T},
\end{aligned}
$$

which is (17).

Equations (19) and (21) can also be given similar to (18) and (20). Equation (23) can be given similar to (22) in (27).

According to the projection theorem, we have

$$
\begin{aligned}
& \widehat{\overline{\mathscr{V}}}_{1}\left(t_{d}+1,2\right) \\
&=\operatorname{Proj}\left\{\overline{\mathscr{V}}_{1}\left(t_{d}+1\right) \mid \widetilde{\mathscr{Y}}_{2}(0), \ldots, \widetilde{\mathscr{Y}}_{2}\left(t_{d}\right)\right\} \\
&=\operatorname{Proj}\left\{\overline{\mathscr{V}}_{1}\left(t_{d}+1\right) \mid \widetilde{\mathscr{Y}}_{2}(0), \ldots, \widetilde{\mathscr{Y}}_{2}\left(t_{d}-1\right)\right\} \\
&+\operatorname{Proj}\left\{\overline{\mathscr{V}}_{1}\left(t_{d}+1\right) \mid \widetilde{\mathscr{Y}}_{2}\left(t_{d}\right)\right\} \\
&= \widehat{\bar{V}}_{1}\left(t_{d}+1 \mid t_{d}-1,2\right) \\
&+H\left(t_{d}+1, t_{d}, 12\right) Q_{\widetilde{\mathscr{Y}}_{2}}\left(t_{d}\right) \widetilde{\mathscr{Y}}_{2}\left(t_{d}\right)
\end{aligned}
$$

then (24) can be given.

\section{Fixed-Point Smoother $\widehat{\mathbf{x}}(t \mid k)$}

By using projection theorem and reorganized innovation, the fixed-point smoother $\widehat{\mathbf{x}}_{1}(t \mid k, 1)(k=t+1, \ldots, N)$ has been given, then it is time to give the main result of fixed-point smoother $\widehat{\mathbf{x}}(t \mid k)$.
Theorem 7. Consider the descriptor system (1) under Assumptions 1 and 2 and $\left[\begin{array}{ll}0 & I\end{array}\right] J_{2}^{-1} \mathbf{x}(0)=-\sum_{j=0}^{\lambda-1} F_{1}^{j} B_{2} \mathbf{u}(j)$; then the optimal fixed-point smoother $\widehat{\mathbf{x}}(t \mid k)=\widehat{\mathbf{x}}(t \mid k, 1)$ can be given by

$$
\widehat{\mathbf{x}}(t \mid k)=J_{2}\left[\begin{array}{l}
\widehat{\mathbf{x}}_{1}(t \mid k, 1) \\
\widehat{\mathbf{x}}_{2}(t \mid k, 1)
\end{array}\right], \quad k=t+1, \ldots, N, t \geq d
$$

In the above, $\widehat{\mathbf{x}}_{1}(t \mid k, 1)$ is from $(14) ; \widehat{\mathbf{x}}_{2}(t \mid k, 1)$ is given as

$$
\begin{aligned}
& \widehat{\mathbf{x}}_{2}(t \mid k, 1) \\
& =\left\{\begin{array}{l}
-\sum_{j=0}^{\lambda-1} F_{1}^{j} B_{2} \widehat{\mathbf{u}}(t+j \mid k, 1), \quad k>t+\lambda-1, \\
-\sum_{j=0}^{k-t} F_{1}^{j} B_{2} \widehat{\mathbf{u}}(t+j \mid k, 1), \quad t<k \leq t+\lambda-1,
\end{array}\right. \\
& \widehat{\mathbf{u}}(t+j \mid k, 1) \\
& =\widehat{\mathbf{u}}(t+j \mid t+j, 1) \\
& +\sum_{i=1}^{k-t-j}\left[G_{1}(t+j, t+j+i, 1) \mathscr{C}_{11}^{T}+T(t+j, t+j+i, 1)\right.
\end{aligned}
$$

where

$$
\begin{aligned}
\widehat{\mathbf{u}}(t+ & j \mid t+j, 1) \\
= & \widehat{\mathbf{u}}(t+j \mid t+j-1,1) \\
& +G(t+j, t+j, 1) Q_{\widetilde{\mathscr{Y}}_{1}}^{-}(t+j) \widetilde{\mathscr{Y}}_{1}(t+j),
\end{aligned}
$$

with $\widehat{\mathbf{u}}\left(t+j \mid t_{d}, 1\right)=\widehat{\mathbf{u}}\left(t+j \mid t_{d}, 2\right)$ being given as

$$
\begin{aligned}
\widehat{\mathbf{u}}\left(t+j \mid t_{d}, 2\right)= & \widehat{\mathbf{u}}\left(t+j \mid t_{d}-1,2\right) \\
& +G\left(t+j, t_{d}, 2\right) Q_{\widetilde{\mathscr{Y}}_{2}}^{-}\left(t_{d}\right) \widetilde{\mathscr{Y}}_{2}\left(t_{d}\right),
\end{aligned}
$$

with

$$
\widetilde{\mathscr{Y}}_{i}(t)=\mathscr{Y}_{i}(t)-\mathscr{C}_{1 i} \widehat{\mathbf{x}}_{1}(t, i)+\widehat{\overline{\mathscr{V}}}_{i}(t, i), \quad i=1,2
$$

and $G(t+j, t+j, 1), G_{1}(t+j, t+j+i, 1), G_{2}(t+j, t+j+i, 1)$, $G\left(t+j, t_{d}, 2\right)$, and $T(t+j, t+j+i, 1)$ can be referred to [19]; $\widehat{\mathbf{x}}_{1}(t, 1)$ is from (18); $\widehat{\mathscr{V}}_{1}(t, 1)$ is from (22).

Proof. Equation (41) can be given easily by considering Lemma 4. 
According to the projection theorem, when $k>t+\lambda-1$, we have

$$
\begin{aligned}
& \widehat{\mathbf{x}}_{2}(t \mid k, 1) \\
& =\operatorname{Proj}\left\{\mathbf{x}_{2}(t) \mid \widetilde{\mathscr{Y}}_{2}(0), \ldots, \widetilde{\mathscr{Y}}_{2}\left(t_{d}\right) ;\right. \\
& \left.\widetilde{\mathscr{Y}}_{1}\left(t_{d}+1\right), \ldots, \widetilde{\mathscr{Y}}_{1}(k)\right\} \\
& =\operatorname{Proj}\left\{-\sum_{j=0}^{\lambda-1} F_{1}^{j} B_{2} \mathbf{u}(t+j) \mid \widetilde{\mathscr{Y}}_{2}(0), \ldots, \widetilde{\mathscr{Y}}_{2}\left(t_{d}\right) ;\right. \\
& \left.\widetilde{\mathscr{Y}}_{1}\left(t_{d}+1\right), \ldots, \widetilde{\mathscr{Y}}_{1}(k)\right\} \\
& =-\sum_{j=0}^{\lambda-1} F_{1}^{j} B_{2} \widehat{\mathbf{u}}(t+j \mid k, 1) .
\end{aligned}
$$

When $t<k \leq t+\lambda-1$, we have

$$
\begin{aligned}
& \widehat{\mathbf{x}}_{2}(t \mid k, 1) \\
& =\operatorname{Proj}\left\{\mathbf{x}_{2}(t) \mid \widetilde{\mathscr{Y}}_{2}(0), \ldots, \widetilde{\mathscr{Y}}_{2}\left(t_{d}\right) ;\right. \\
& \left.\widetilde{\mathscr{Y}}_{1}\left(t_{d}+1\right), \ldots, \widetilde{\mathscr{Y}}_{1}(k)\right\} \\
& =\operatorname{Proj}\left\{-\sum_{j=0}^{k-t} F_{1}^{j} B_{2} \mathbf{u}(t+j) \mid \widetilde{\mathscr{Y}}_{2}(0), \ldots, \widetilde{\mathscr{Y}}_{2}\left(t_{d}\right) ;\right. \\
& \left.\widetilde{\mathscr{Y}}_{1}\left(t_{d}+1\right), \ldots, \widetilde{\mathscr{Y}}_{1}(k)\right\} \\
& =-\sum_{j=0}^{k-t} F_{1}^{j} B_{2} \widehat{\mathbf{u}}(t+j \mid k, 1) ;
\end{aligned}
$$

then (47) and (48) yield (42).

According to the projection theorem, we have

$$
\begin{aligned}
& \widehat{\mathbf{u}}(t+j \mid k, 1) \\
& =\operatorname{Proj}\left\{\mathbf{u}(t+j) \mid \widetilde{\mathscr{Y}}_{2}(0), \ldots, \widetilde{\mathscr{Y}}_{2}\left(t_{d}\right) ;\right. \\
& \left.\widetilde{\mathscr{Y}}_{1}\left(t_{d}+1\right), \ldots, \widetilde{\mathscr{Y}}_{1}(k)\right\} \\
& =\operatorname{Proj}\left\{\mathbf{u}(t+j) \mid \widetilde{\mathscr{Y}}_{2}(0), \ldots, \widetilde{\mathscr{Y}}_{2}\left(t_{d}\right) ;\right. \\
& \left.\widetilde{\mathscr{Y}}_{1}\left(t_{d}+1\right), \ldots, \widetilde{\mathscr{Y}}_{1}(t+j)\right\} \\
& +\sum_{i=1}^{k-t-j} \operatorname{Proj}\left\{\mathbf{u}(t+j) \mid \widetilde{\mathscr{Y}}_{1}(t+j+i)\right\}
\end{aligned}
$$

$$
\begin{aligned}
& =\widehat{\mathbf{u}}(t+j \mid t+j, 1) \\
& +\sum_{i=1}^{k-t-j}\left\langle\mathbf{u}(t+j), \widetilde{\mathscr{Y}}_{1}(t+j+i)\right\rangle \\
& \cdot Q_{\widetilde{\mathscr{Y}}_{1}}^{-}(t+j+i) \widetilde{\mathscr{Y}}_{1}(t+j+i) \\
& =\widehat{\mathbf{u}}(t+j \mid t+j, 1) \\
& +\sum_{i=1}^{k-t-j}\left[G_{1}(t+j, t+j+i, 1) \mathscr{C}_{11}^{T}+T(t+j, t+j+i, 1)\right. \\
& \left.\quad-G_{2}(t+j, t+j+i \mid t+j+i-1,1)\right] \\
& \cdot Q_{\widetilde{\mathscr{Y}}_{1}}^{-}(t+j+i) \widetilde{\mathscr{Y}}_{1}(t+j+i),
\end{aligned}
$$

which is (43).

Equations (44) and (45) can be given similar to (20).

\section{Conclusion}

The optimal fixed-point smoother for the descriptor system has been proposed, where the system model is corrupted by multiplicative noise, and the system is observed by instantaneous and single delayed observations. By using standard singular value decomposition, the origin system has been changed into the restricted system, fixed-point smoother is given based on reorganized innovation analysis [17] and projection theorem for the restricted system model, and then the fixed-point smoother for the origin system has been given based on the above result. The fixed-point smoother is given in terms of recursive Riccati equation and can be computed easily [21].

\section{Conflict of Interests}

The authors declare that there is no conflict of interests regarding the publication of this paper.

\section{Acknowledgments}

This paper is supported by the National Nature Science Foundation of China (61273197), Nature Science Foundation of Shandong Province (ZR2013FM018), the Science Research Foundation for the Excellent Middle-Aged and Youth Scientists of Shandong Province (BS2013DX012), the Applied Fundamental Research of Qingdao (14-2-4-19-jch), Huangdao District Science and Technology Project (2014-133), and "Taishan Scholarship" Construction Engineering.

\section{References}

[1] N. Wiener, Extrapolation Interpolation, and Smoothing of Stationary Time Series, The Technology Press and Wiley, New York, NY, USA, 1949.

[2] R. E. Kalman, "A new approach to linear filtering and prediction problems," Journal of Basic Engineering, vol. 82, no. 1, pp. 35-45, 1960. 
[3] T. Kailath, A. H. Sayed, and B. Hassibi, Linear Estimation, Prentice Hall, Englewood Cliffs, NJ, USA, 1999.

[4] B. D. O. Anderson and J. B. Moore, Optimal Filtering, Prentice Hall, Englewood Cliffs, NJ, USA, 1979.

[5] Y. J. Liu, C. L. P. Chen, G. X. Wen, and S. Tong, "Adaptive neural output feedback tracking control for a class of uncertain discrete-time nonlinear systems," IEEE Transactions on Neural Networks, vol. 22, no. 7, pp. 1162-1167, 2011.

[6] Y. Liu and S. Tong, "Adaptive neural network tracking control of uncertain nonlinear discrete-time systems with nonaffine deadzone input," IEEE Transactions on Cybernetics, vol. 45, no. 3, pp. 497-505, 2015.

[7] Y.-J. Liu, L. Tang, S. Tong, C. L. P. Chen, and D.-J. Li, "Reinforcement learning design-based adaptive tracking control with less learning parameters for nonlinear discrete-time MIMO systems," IEEE Transactions on Neural Networks and Learning Systems, vol. 26, no. 1, pp. 165-176, 2015.

[8] H. Gao, T. Chen, and T. Chai, "Passivity and passification for networked control systems," SIAM Journal on Control and Optimization, vol. 46, no. 4, pp. 1299-1322, 2007.

[9] H. Zhang, L. Xie, and Y. C. Soh, "Risk-sensitive filtering, prediction and smoothing for discrete-time singular systems," Automatica, vol. 39, no. 1, pp. 57-66, 2003.

[10] H. S. Zhang, T. Y. Chai, and X. J. Liu, "A unified approach to optimal state estimation for stochastic singular systems," Automatica, vol. 34, no. 6, pp. 777-781, 1998.

[11] L. Y. Dai, "Filtering and LQG problems for discrete-time stochastic singular systems," IEEE Transactions on Automatic Control, vol. 34, no. 10, pp. 1105-1108, 1989.

[12] S. Nakamori, R. Caballero-guila, A. Hermoso-Carazo, and J. Linares-Prez, "Design of recursive Wiener fixed-point smoothers based on innovations approach in linear discretetime stochastic systems," Applied Mathematics and Computation, vol. 165, no. 3, pp. 731-747, 2005.

[13] S. Nakamori, "Design of extended recursive Wiener fixed-point smoother and filter in discrete-time stochastic systems," Digital Signal Processing, vol. 17, no. 1, pp. 360-370, 2007.

[14] D. Chu and S. Gao, "State optimal estimation algorithm for singular systems with multiplicative noise," Periodical of Ocean University of China, vol. 38, no. 9, pp. 814-818, 2008.

[15] D. Chu, S. Gao, and L. Guo, "State estimation for multi-channel stochastic singular systems with multiplicative noise," Asian Journal of Control, vol. 12, no. 6, pp. 725-733, 2010.

[16] S. Gao, Study of optimal estimation algorithm for singular systems with multiplicative noise [M.S. thesis], Ocean University of China, 2010.

[17] X. Lu, X. Dong, and H. Wang, "Optimal fixed-point smoothing for delayed descriptor systems with multiplicative noise," in Proceedings of the 27th Chinese Control and Decision Conference, Qingdao, China, 2015.

[18] H. Zhang, L. Xie, D. Zhang, and Y. C. Soh, "A reorganized innovation approach to linear estimation," IEEE Transactions on Automatic Control, vol. 49, no. 10, pp. 1810-1814, 2004.

[19] X. Lu, H. Wang, and F. Wu, “Optimal multiple-step predictor for singular systems with multiplicative noise and delayed measurement," Control Theory \& Applications. Revised.

[20] H.-Q. Wang, H.-S. Zhang, G.-R. Duan, and G.-C. Wang, "Design of H-infinity multi-step predictor for descriptor systems," Journal of Control Theory \& Applications, vol. 24, no. 5, pp. 693-700, 2007.
[21] K. M. Nagpal and P. P. Khargonekar, "Filtering and smoothing in an $H_{\infty}$ setting," IEEE Transactions on Automatic Control, vol. 36, no. 2, pp. 152-166, 1991. 


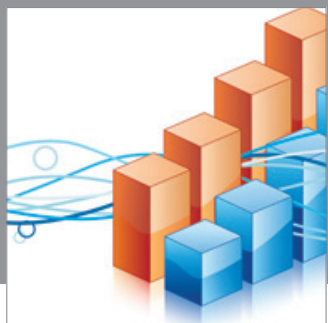

Advances in

Operations Research

mansans

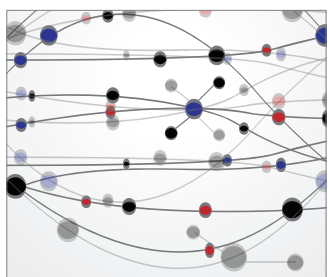

The Scientific World Journal
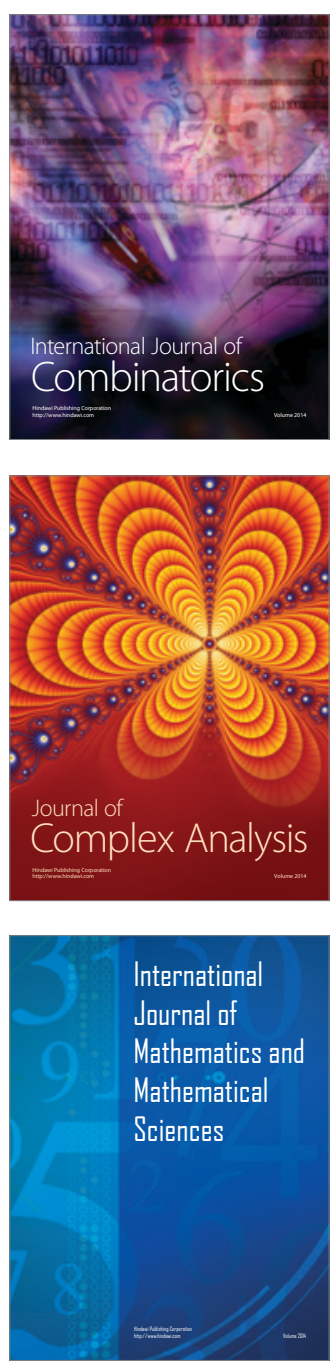
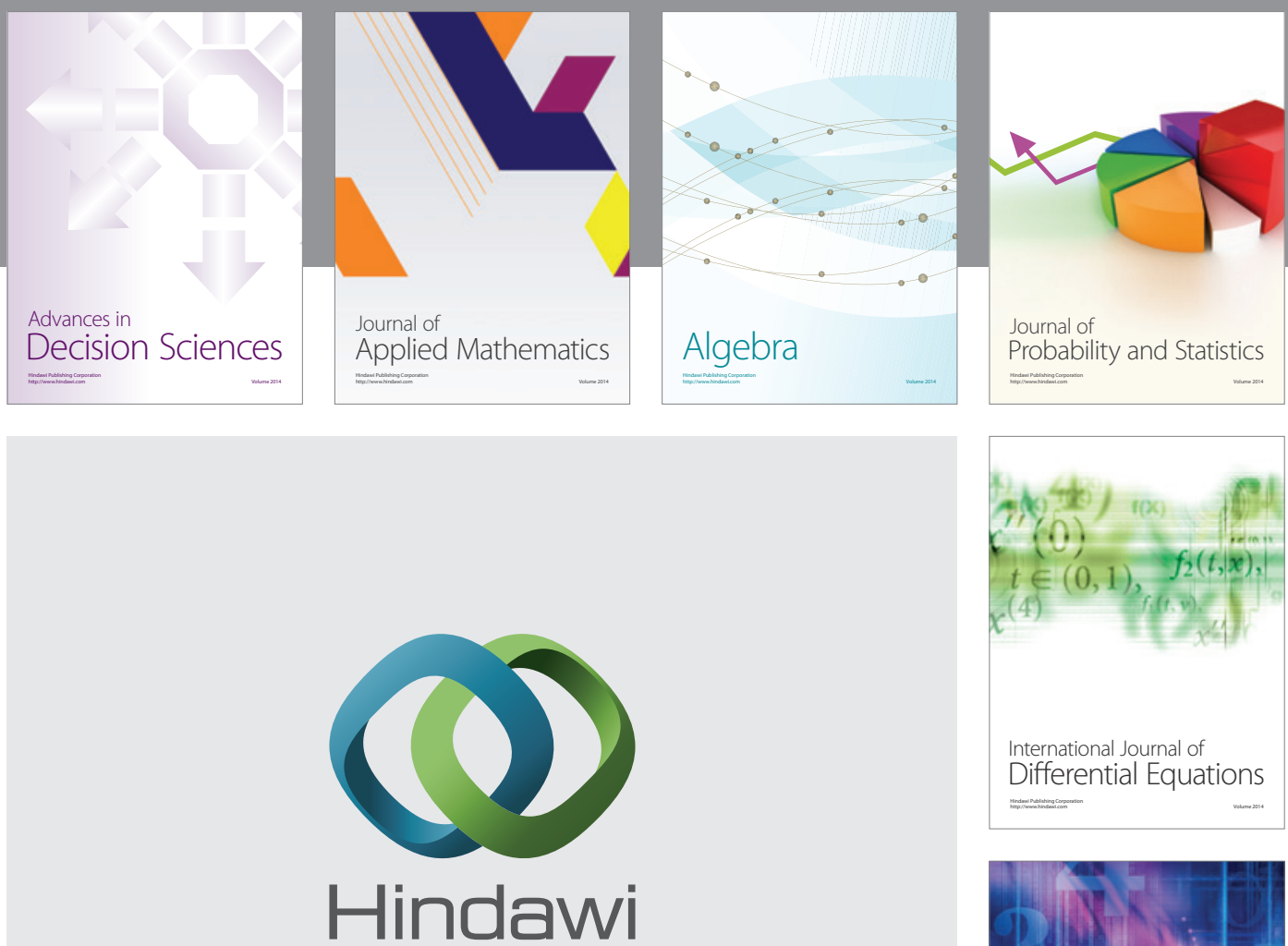

Submit your manuscripts at http://www.hindawi.com
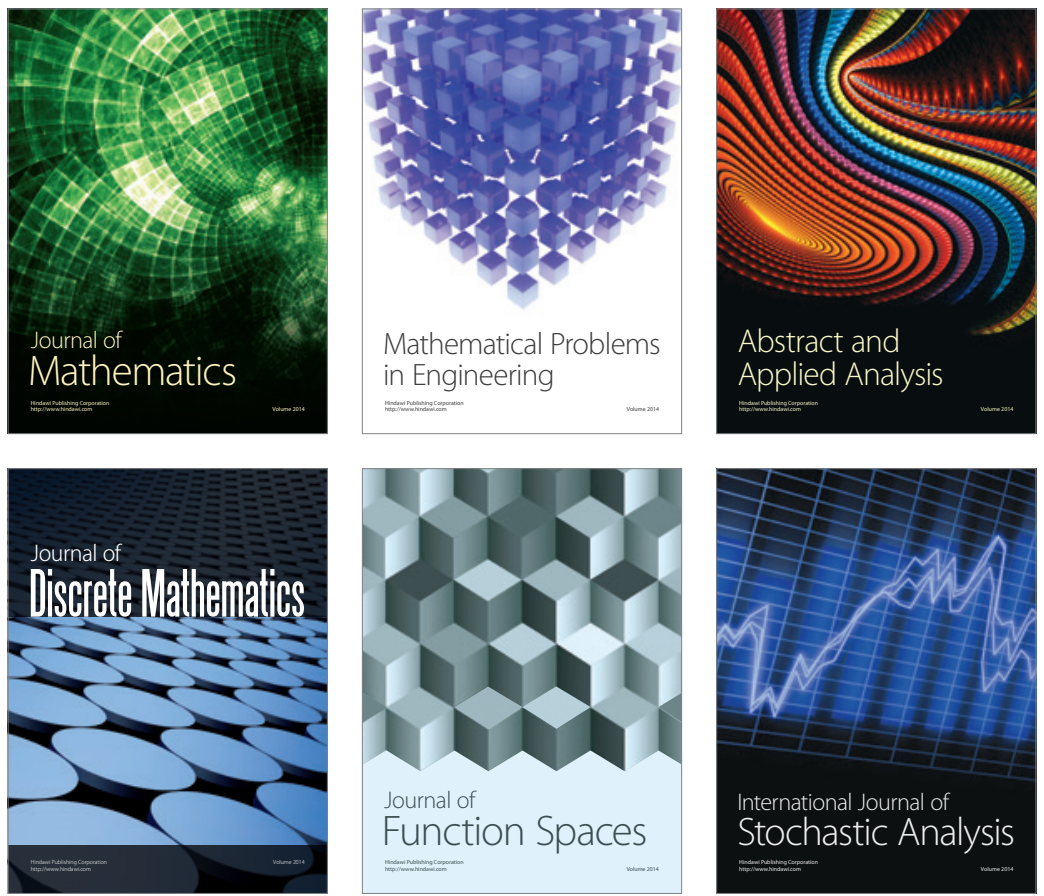

Journal of

Function Spaces

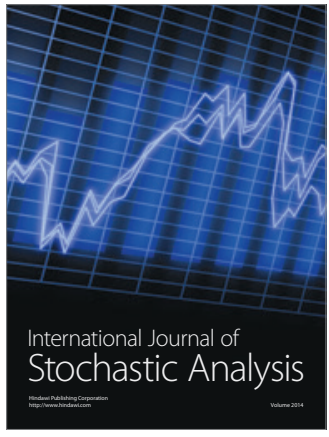

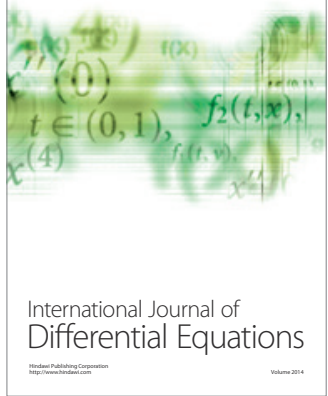
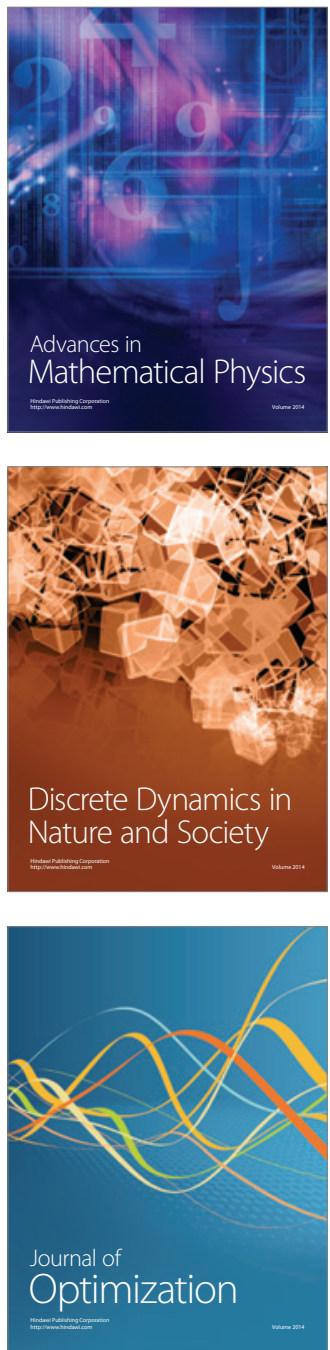